\title{
Decoding of Akhlat/Mezadj (A/M) or Humor/Temperament (H/T) theory For Mucu-Cataneous Infectious Diseases in Persian Medicine
}

\author{
Running Title: Mucous-Cutaneous Infectious Diseases in Persian Medicine \\ Majid Avijgan $^{1^{*}}$, Maryam Ansari ${ }^{2}$, Firoozeh Salhzadeh ${ }^{3}$ \\ ${ }^{I}$ Professor of Infectious Diseases and Traditional Persian Medicine, Isfahan University of Medical Sciences, \\ Iran \\ ${ }^{2}$ Resident ship of Infectious Diseases, Isfahan University of Medical Sciences, Iran \\ ${ }^{3}$ Resident ship of OBGY, Isfahan University of Medical Sciences, Iran
}

*Corresponding Author: Majid Avijgan, P.O. Box 795, Alzahra hospital, Soffe St, Isfahan, Iran, Email: avijgan@yaho.com

Keywords: Akhlat Mezadj Theory, Persian Medicine, Skin, Mucous membrane, Infectious Diseases, Ludwig angina, TB, Leprosy

\section{INTRODUCTION}

This review is retrieved from doctorate thesis (1) about Infectious Diseases (ID) described in ancient text book in Persian Medicine (PM) named TIB-E-AKBARI which is essential of two previous and ancient text book of PM; 1SHARH OL ASBAB written by NAFIS IBN EIVAZ-E-KERMANY and 2-ASBAB va ALA,EM written by SAMARGHANDI in about 700 years ago. TIB-E-AKBARI which is one of the famous written books of PM, includes a detail description of nearly 100 of common of Infectious Disease (ID). In a recent comparative study these one hundred of ID have decoded and became evidence base (1). As a new finding of this report, the Infectious Diseases are called and described not as by etiologic agents as modern medicine but based on PM's terminology as Humor which must be cleared and decoded. This report is trying to decode the meaning of term of Humor or etiologic agents in $\mathrm{PM}$ viewpoint.

\section{Humor AS FOUNDATION OF MEZADJ (TEMPERAMENT)}

Humor is made of food, drinking and whatever entered into the body via inhalation or by penetration into skin. Humor will be an issue which is in constant movement (in human body) and are the base of healthy life. According to PM there are four symbolic humors in human body in the form of gaseous (Choleric humor), thick liquid or water (Phlegmatic humor), thin liquid or water (Blood humor) and substance (Melancholic humor). These are Quadruple Humors in PM.

These quadruple humor are final products of whatever entering into the body which have to used and have a constant movement in a circulating manner; Blood (thin liquid or water) in the blood vessel, Phlegm (thick liquid or water) in Lymphatic pathway and connective tissue. Yellow bile or Choleric humor is as ROUH/QI/Energy which is circulating in the PVS (2). Black bile or Melancholic humor as substance or material can be similar to circulating (abnormal) cells (such as cancerous cells) and or abnormal micro or macro molecules in human body, for example low molecular weight protein (3) or Waldenström macroglobulinemia (4). These quadruple Humors have qualities by which can make the native Mezadj (Temperament) in normal condition but in abnormal condition can affect and change the native Mezadj (Temperament) IZheng(5) to So-e-Mezadj (Dystemperament).

\section{What is humor?}

\section{Decoding of Humor in TPM}

\section{Yellow Bile or Choleric Humor}

It has the qualities of Heat and Dry, similar to fire, invisible state, penetrating into every hole 
of body and transmitting the heat and dryness to penetrated place. For example acute bacterial and or viral infection can resulted from choleric humor. Its color is yellow, orange and or red (similar to pus production in ID). Similarly the color of face in acute infection will be reddish.

\section{Blood Humor}

It has the qualities of Heat and Wetness, similar to air, by which lead to increasing the heat and wetness of penetrated place so that swelling such as inflammation can be resulted from a blood humor. Its color is red. The connective tissue disorders with various inflammations can be classified in relation to the disorder of this humor in PM.

\section{Phlegmatic Humor}

It has qualities of Cold and wetness, similar to water or liquid and by having a fluid feature has a directed and constant movement which in the case of any pathology in this movement leads to diseases. Stasis or "stop-motion" of water can be leading to the pathology of water, such as swamps, marshes. For example pleural effusion, pericardial effusion, obesity can be described by pathology of phlegmatic humor. Its color is withe.

\section{Melancholic Humor}

It has qualities of Cold and Dryness, similar to earth, and in the case of any pathology in the movement, by having the gravity, intends to stasis or stop-motion.

Diseases related to Melancholic Humor are presented by disfiguration of tissue, such as cancerous diseases and or by deposition of Humor(cancerous cells and abnormal molecules) in the skin such as diabetic foot ulcer (or eczema and dermatitis) and or caused by impairing of the blood and lymphatic circulation leads to stasis and therefore chronic disease like chronic bed sores. Its color is balck.

Any of these Humor have a stable amount in human body (in healthy state), so that in the case of increasing or decreasing in quantity and quality features of Humor, disease will appear.

In other words Humor which is a product of what entered into the body, has a specific and characteristic quality per se and has a general effect on human body. The general effect of Humor will be appeared after their distribution into body.

Then there will be a physiologic Humor which is used for optimal level of living and normal function and the second one as pathologic
Humor (pathogen, toxin.....) which is harmful for living and lead to dysfunction or disease. Any way both of them will enter into the body via eating, drinking or through skin and inhalation.

The inherited genes and chromosomes will determine the native Temperament or Zheng/Mezadj (5) of human which after birth, Quadruple Humor (coming from food, drink, inhalation, and penetration and so) will maintain the Temperament human body in normal state of health or abnormal state or dystemperament (SoeMezadj or Bad Mezadj).

Based on presented Akhlat/Mezadj (A/M) theory or Humor/Temperament (H/T) theory, quadruple humor including Choleric, phlegmatic, Bloody and Melancholic Humors are used for understanding most of the ID.

The some common diseases in the field of ID will be presented in following section and be discussed in details.

\section{A/M or $\mathbf{H} / \mathbf{T}$ Theory (1)}

The history of PM indicates that ID are one of the most common diseases among the diseases of ancient time. In PM view point, etiology of ID have been described and managed by several terminology which the famous one is Akhlat/ Mezadj (A/M) theory or Humor/ Temperament $(\mathrm{H} / \mathrm{T})$ theory.

Akhlateh and Mezadj (A/M) theory is major idea for describing terminology of ID in PM by which most of the infectious diseases have been explained. Although scholars (Hakim in PM) (6) didn't have any knowledge about epidemiology and transmission of ID but nevertheless in at least one of the ID, Rabies the transmission of etiologic agent, from dog to human, has been indicated and showing a knowledge behind the prominent theory. They have strongly recommended avoidence from any contact with infected $\operatorname{dog}$ so that this recommendation remembers the zoonosis transmission of Rabies.

\section{KHONAG(pharyngo-laryngo- tranchobronchitis) as a Dystemperament (Bad Mezadj) (1)}

In other side, some other of ID has been described based on So-e-Mezadj (Dystemperament) for example in $\boldsymbol{K H O N A G}$ (pharyngo-laryngo-tranchobronchitis) that can be manifested due to Dystemperament of Choleric, phlegmatic, Sanguine (Bloody) and Melancholic humor. Given that decoding of this study, Bloody Sanguine Dystemperament can be decoded as viral pharyngitis in which main 
manifestation is hyperemia of nasopharynx. In addition, Choleric Dystemperament can be bacterial angina (like streptococcal angina with acute, short course severe manifestation), Epstein Barr Virus angina with insidious course and sever swelling in tonsillar can be similar to Phlegmatic Dystemperament and finally nasopharyngeal and laryngeal cancerous state could be a state of Melancholic Dystemperament.

\section{ZAAT OL JANB (Pleural Effusion) by Quadruple Humor (1)}

There are four types of ZAAT OL JANB or Pleural effusion (PE) as Pure, Pure Choleric, Pure Phlegmatic and Melancholic. According to this theory, decoding by study indicates that Pure type can be replaced by transudate pleural effusion, Pure Choleric can be exudative PE, Pure phlegmatic can be chronic PE (for example TB) and Melancholic PE due to primary and secondary cancerous tissue and.

\section{Infected or Putrefied Material (1)}

The other type of description for ID use is neither of above description and is showing infected material or putrefied material as etiologic agent of some of ID.

It seems that scholars (Hokama) of PM for description of these category of ID used neither $\mathrm{A} / \mathrm{M}$ theory nor invasive Quadruple human in the body. Nevertheless they knew that abnormal materials or subjects are added to human body. Intestinal parasite and Dracunculus medinensis ( a tropical skin infection) are categorized in this type of etiologic agent. FAZ, LEH-e-RADDIEH (they believed it as inappropriate humor) which is a term for description of burnt Phlegm or burnt Melancholic Humors are the etiologic agent of these later infectious disease.

The difference between last mentioned theory and other theories is that in $\mathrm{A} / \mathrm{M}$ theory, normal quadruple humors can lead to disease but base on last theory an abnormal Humor such as burnt Phlegm or burnt Melancholic Humors can lead to dracunculus medinensis or intestinal parasite.

Another example of this kind of disease is Ludwig angina or (ZAHABE) which is presented by Humor of warm thick and putrefied blood. Also chronic prostatitis is due to thick and slimy Humor or Vincent's angina (AKELAT Ol FAM) is presented by infected and slimy Humor.

In all of those examples, description of ideas are because there is increased (transformed or changed) and abnormal Humor in human body.

\section{Change on Quality: Warm dystemperament or Warm Inflammation}

By considering these methods of decoding it can be understood that some terminologies have been used for other description of ID. For example one of kidney disorder is Warm Inflammation of Kidney. Basically the temperament of kidney and urinary tract is cold and wet (1). In the case of change in kidney temperament from cold (normal) to warm (abnormal) feature (due to hyperemia) is called Warm Inflammation.

In other side, warm is the quality of Choleric Humor, based on this decoding, Choleric Humorare expressed as acute bacterial agent so that Warm Dystemperament (SoeMezadj) of kidney can be translated in to UTI due to bacterial agent.

In other word,warm inflammation of kidney is another disorder which is different from Warm Dystemperament (SoeMezadj) of kidney. Warm inflammation of kidney probably means nephritis. There is mainly swelling and hyperemia which are the characteristic of Sanguine Humor, in this case warm inflammation of kidney can be decoding in to acute pyelonephritis.

\section{Other Description}

\section{TB}

The word of tuberculosis has been repeatedly mentioned in the text book of TIB-e-AKBARI in $\mathrm{PM}$ which in all of these diseases, TB (cell in $P M$ ) does not mean actuall tuberculosis.

Issue of tuberculosis is described neither by AKHLAT/Mezadj theory, nor putrefied infected material nor by change of quality of temperament.

The word of TB seems that is unique category and is used for description of Anaerobic, gram negative and gram positive bacteria such as staphylococcal bacterial pneumonia in lung and also skin infections by atypical mycobacteriums.

It seems that scholars of PM have been interested in autopsy so that in postmortem autopsy any cavitation of lung was related to TB. In this case, all of the acute necrotizing pneumonia, wrongly, are categorized in the TB disease.

\section{Leprosy}

The topic of leprosy, probably it seems that is one of the incorrect imagination of scholars, although their opinion about clinical 
manifestation of leprosy is admirable, and they have noted to details of leprosy (1).

In TIB-e-AKBARI medicine textbook, there are two describing of clinical types of leprosy, Pauci-bacillary and Multi bacillary clinical presentation which have difference clinical pattern and have written in details as good as modern medicine.

Melancholic Humor is presented as the etiologic agent of leprosy (in PM) and in other side by decoding of Melancholic Humor for leprosy will be indicated that there is not any incorrect definition. Melancholic Humor is used for presentation of end stage of diseases or cancerous state, in other side leprosy is accompanied with micro-necrosis of extremities and cold region of body such as fingers and nose. Final clinical manifestation of leprosy which is accompanied with necrosis is similar to cancerous state and chronic ulcers. Clearly the etiologic agent of leprosy can be presented as Melancholic humor (1).

\section{CONCLUSION}

Based on decoding theory of this study, in AKHLAT (Humor)/ MEZADJ (Dystemperament) theory of PM can be defined:

1. Choleric Humor (in most of the cases) means acute Gram negative, Gram positive and anaerobic infection of skin or systemic.

2. Sanguine (Bloody) Humor which presents hyperemia, tissue vasodilation, redness and swelling of mucous membrane, in most cases means viral infection and sometimes bacterial agent.

3. Phlegmatic Humor means infective etiologic agent with chronic and insidious course such as mycobacterial and fungal infection in mucous-cutaneous and systemic infections.

4. Melancholic Humor indicates chronic tissue and dermatomal necrosis (sometimes acutely) which are mainly due to cancers and sometimes infectious disease. By this idea all diabetic foot ulcers can be categorised in the melancholic disorders.

This method of decoding can facilitate reading of the texts of PM and there is new horizon for researcher in ID field in PM. By this new idea reviewing in diagnostic, therapeutic and follow up methods of patients in PM, can suggest new insight of complementary method in modern medicine. This conclusion can be very useful especially in infections with antibiotic resistance pattern which is very useful for next generations of researchers.

\section{REFERENCES}

[1] Salehzadeh Firoozeh, Avijgan Majid, Mazaheri Mohhamad. Comparison of Clinical Finding of common Infectious Diseases in AKBARAI $t$ extbook of Persian Medicine and Modern Medi cine (Harrison 2015). Isfahan University of Medical Sciences.2016 Spetember.As Thesis for doctorate Graduation. Identification Number of Thesis: 395074)

[2] Avijgan M, Avijgan M. Can the primo vascular system (Bong Han Duct System) be a basic concept for Qi production? International Journal of Integrative Medicine. 2013;1(20): $1-10$.

[3] Okada N, Aizawa T, Yokokawa N, Kobayashi M, Moriya $\quad T$, Shigematsu S, Shirota T, Komatsu M, Shinoda T, Yamada T, et al.Abnormal molecular weight profile of urinary protein in rats with streptozotocininduced diabetes.Diabetes Res ClinPract. 1992 Oct;18(1):1-9.

[4] Gertz MA. Waldenströmmacroglobulinemia: 2013 update on diagnosis, risk stratification, and management. Am J Hematol. 2013;88:703711.

[5] Avijgan M, Avijgan M. The Infrastructure of the Integrative Human Body; Qi/Dameh, Qi Movement/Rouh and Zheng/Mezadj; Scientific Base. International Journal of Integrative Medicine. 2013; 1 (22): 2013

[6] STANLEY B. BURNS, STEPHEN FULDER Arabic Medicine: Preservation and Promotion. A Millennium of Achievement. THE JOURNAL OF ALTERNATIVE AND COMPLEMENTARY MEDICINE Volume 8, Number 4, 2002, pp. 407- 410

Citation: Majid Avijgan, Maryam Ansari, Firoozeh Salhzadeh. Decoding of Akhlat/Mezadj (A/M) or Humor/Temperament (H/T) theory. ARC Journal of Dermatology. 2018; 3(1):6-9. doi:dx.doi.org/10.20431/24560022.0301003.

Copyright: () 2018 Authors. This is an open-access article distributed under the terms of the Creative Commons Attribution License, which permits unrestricted use, distribution, and reproduction in any medium, provided the original author and source are credited. 